\title{
Temporal Innovization: Evolution of Design Principles Using Multi-objective Optimization
}

\author{
Sunith Bandaru ${ }^{a}$ and Kalyanmoy Deb $^{b}$ \\ ${ }^{a}$ Virtual Systems Research Centre, University of Skövde, Skövde, Sweden \\ sunith.bandaru@his.se \\ ${ }^{b}$ Department of Electrical and Computer Engineering, \\ Michigan State University, USA \\ kdeb@egr.msu.edu
}

COIN Report Number 2014016

\begin{abstract}
Multi-objective optimization yields multiple solutions each of which is no better or worse than the others when the objectives are conflicting. These solutions lie on the Pareto-optimal front which is a lower-dimensional slice of the objective space. Together, the solutions may possess special properties that make them optimal over other feasible solutions. Innovization is the process of extracting such special properties (or design principles) from a trade-off dataset in the form of mathematical relationships between the variables and objective functions. In this paper, we deal with a closely related concept called temporal innovization. While innovization concerns the design principles obtained from the trade-off front, temporal innovization refers to the evolution of these design principles during the optimization process. Our study indicates that not only do different design principles evolve at different rates, but that they start evolving at different times. We illustrate temporal innovization using several examples.
\end{abstract}

\section{Introduction}

Evolutionary algorithms (EAs) are ideal for multi-objective optimization problems since they evolve a population of randomly initialized solutions by iteratively applying operators that mimic the natural evolution process and converge to a set of near Pareto-optimal solutions (or trade-off solutions) all of which are high-performing with respect to the conflicting objectives. These Pareto-optimal solutions are special in some sense because they lie on a lower-dimensional manifold of the objective space. It is therefore natural to assume that they may possess exclusive properties which make them Pareto-optimal. While analytically deriving such properties from the optimization problem may not always be possible, an alternate approach is to first obtain a representative trade-off dataset of (near) Pareto-optimal solutions using an MOEA and then apply machine learning techniques to extract mathematical relationships that are valid on either a part or whole of the dataset. Since the trade-off dataset usually contains columns corresponding to the variable values and the corresponding function values, the extracted relationships depict correlations between these entities. 
In our past works we have shown that such mathematical properties do exist and can be obtained from the trade-off dataset through a process called innovization - innovation through optimization. The original innovization methodology [7] involved manually plotting various combinations of the columns in the tradeoff datasets, visually identifying correlations and using mathematical functions to perform regression on the correlated parts of the dataset. The method was tedious and prone to errors. However, our recent works $[1,8]$ have dealt with automating the innovization process by using clustering methods to automatically identify the correlations. This new automated innovization approach is capable of generating multiple significant relationships and has been successfully applied to practical engineering design problems. The obtained relationships are referred to as design principles because they are extremely useful for the designer in understanding how different variables should vary for maintaining Pareto-optimal operation/performance of the system or design.

\subsection{Temporal Innovization and Human Evolution}

Trade-off solutions are the end result of an MOEA and hence the design principles obtained through automated innovization pertain to the final generation of the MOEA. However, all MOEAs start with a randomly initialized population of solutions which are evolved using operators that, to some extent, mimic the natural process of evolution over several generations. Ignoring complex phenomena such as dynamic environments, cooperative individuals, sexual reproduction and interspecies interactions, the evolutionary optimization process can be viewed similar to the natural process of human evolution.

Homo sapiens acquired various anthropological features during the process of human evolution. There is sufficient documented evidence showing that these features evolved gradually over millions of years [11], rather than appearing out as a single event, driven by the natural mechanisms of reproduction, genetic mutation and natural selection. Despite the relative simplicity of MOEAs, the design principles that Pareto-optimal solutions possess can be thought of as somewhat analogous to the anthropological features. Just as the anthropological features distinguish present day humans and make them high-performing when compared to their ancestors, the design principles make Pareto-optimal solutions high-performing among all other feasible solutions.

Temporal innovization refers to the study of evolution of design principles over generations of an MOEA. The detailed procedure was laid out in [5]. The goal of this paper is to perform temporal innovization on various engineering design problems in order to support and extend the results provided in [5]. Specifically, we investigate if there exists a gradual evolution of design principles over generations of an MOEA, in the same way that anthropological features of humans developed gradually over millions of years. Archaeological evidence also shows that human evolution involved a hierarchy of keys developments. In other words, different anthropological features appeared at different times in the evolutionary time-line. The vertebrae emerged first, followed by the appearance of the first limbs, and so on. Adequate development of certain features was 
essential for some other feature to appear. If design principles indeed resemble the anthropological features, there is reason to believe that they too may exhibit hierarchical evolution in addition to gradual evolution over the generations of an MOEA. In this paper, we also investigate whether such hierarchy occurs in the evolution of design principles.

The paper is organized as follows. In Section 2 we describe the methodology for performing a temporal innovization study and generating the evolution plots. We use this approach on three engineering design problems in Section 3 and interpret the evolution plots. In Section 4, we use a visual method for the extraction of design principles on two topology optimization problems. Temporal innovization is also performed visually.

\section{Methodology}

MOEAs being stochastic by nature, the route that evolving solutions take while converging towards the Pareto-optimal front may differ between runs, even if the trade-off front obtained at the end is approximately the same in all the runs. In order to account for this statistical variance, the first task is to obtain generation-wise population datasets from multiple runs of the same evolutionary algorithm. In this paper we use NSGA-II [4] to solve the multi-objective problem at hand. Multiple runs are executed with uniformly distributed seed values for the random number generator.

One of the trade-off datasets is randomly chosen and the design principles are extracted using the automated innovization algorithm developed in [8]. The details of this algorithm are irrelevant to discussion in this paper. However, it suffices to mention that the design principles obtained have the mathematical form,

$$
\prod_{j=1}^{N} \phi_{j}(\mathbf{x})^{a_{j} b_{j}}=c,
$$

where, $\phi_{j}$ 's are the symbolic entities (variables and objectives functions) called basis functions which can have a Boolean exponent $a_{j}$ and a real valued exponent $b_{j}$. Each design principle is associated with a significance value which indicates the percentage of trade-off solutions for which that design principle remains invariant, i.e. takes a (approximately) constant value $c$.

For recording the evolutionary time-line of design principles, the non-dominated solutions from each of the runs at each generation $t$ are stored. Next, each design principle $(\mathrm{DP} i)$ is checked for its presence in the combined data at each generation. The significance of $\mathrm{DP} i$ at generation $t$, denoted by $S_{t}^{\mathrm{DP} i}$, is calculated as the proportion of points satisfying the design principle to the total non-dominated points in the final generation $(N G E N)$. The stepwise procedure for calculating $S_{t}^{\mathrm{DP} i}$ for a given design principle $\mathrm{DP} i$ is presented below:

Step 0: Set $t \leftarrow 0$.

Step 1: Collect solutions at generation $t$ from all runs into the set $\mathbf{P}_{t}$. Thereafter, remove the dominated points from $\mathbf{P}_{t}$. 
Step 2: Evaluate DP $i$ at all solutions in $\mathbf{P}_{t}$ to obtain $c$-values and collect them in set $\mathbf{C}_{t}$.

Step 3: Every element $c \in \mathbf{C}_{t}$ is checked for its association with any of the $\mathcal{C}$ clusters of $\mathrm{DP} i$ using the criterion,

$$
c \in \text { cluster } k \Leftrightarrow \mu_{c}^{(k)}-s \sigma_{c}^{(k)} \leq c \leq \mu_{c}^{(k)}+s \sigma_{c}^{(k)},
$$

where $\mu_{c}^{(k)}$ and $\sigma_{c}^{(k)}$ are respectively the mean and standard deviation for the $k$-th cluster. The number of elements $E_{t}$ in $\mathbf{C}_{t}$ that belong to any one of the $\mathcal{C}$ clusters is recorded. A variation of $s$ standard deviations is allowed in the $c$-values. In this paper $s=4$ is used and recommended.

Step 4: Calculate the significance of $\mathrm{DP} i$ in the current generation $t$ as,

$$
S_{t}^{\mathrm{DP} i}=\frac{E_{t}}{\left|\mathbf{P}_{N G E N}\right|} \times 100 \%,
$$

where |.| represents the set size.

Step 5: If $t=N G E N$ Stop else $t \leftarrow t+1$ and Goto Step 1.

Thereafter, a plot of the significance value of each design principle with generation is used to reveal the relative order in which design principles appear during the optimization process. The plot also shows which of design principles evolve faster and which ones evolve at a slow rate.

\section{Results I: Design Principles through Automated Innovization}

The procedure described above is now illustrated on three engineering design problems, namely, car side impact problem, metal cutting problem and MEMS resonator design problem. While the first two problems are relatively simple mathematical models of complex design problems, the latter is directly a realworld problem which takes all practical design considerations into account. The difference between the three becomes clear in the following sections.

\subsection{Car Side Impact Problem}

A car is subjected to a regulatory side impact test. Various impact loads, rib deflections and a quantity called viscous criterion $\left(\mathrm{V}^{*} \mathrm{C}\right)$ are measured for the crash test dummy. The velocities of B-pillar midpoint and front door are measured on the vehicle structure. The following decision variables are to be optimized.

$$
\begin{array}{lll}
0.5 & \leq x_{1}: \text { Thickness of B-Pillar inner } & \leq 1.5 \mathrm{~mm} \\
0.45 \leq x_{2}: \text { Thickness of B-Pillar reinforcement } & \leq 1.35 \mathrm{~mm} \\
0.5 \leq x_{3}: \text { Thickness of floor side inner } & \leq 1.5 \mathrm{~mm} \\
0.5 & \leq x_{4}: \text { Thickness of cross members } & \leq 1.5 \mathrm{~mm} \\
0.875 \leq x_{5}: \text { Thickness of door beam } & \leq 2.625 \mathrm{~mm} \\
0.4 \leq x_{6}: \text { Thickness of door beltline reinforcement } & \leq 1.2 \mathrm{~mm} \\
0.4 & \leq x_{7}: \text { Thickness of roof rail } & \leq 1.1 \mathrm{~mm}
\end{array}
$$


Table 1: Design principles for the car side impact problem.

\begin{tabular}{|c|c|c|}
\hline Notation & Design principles & Significance \\
\hline DP1 & $x_{6}^{1.0000}=$ constant & $77.44 \%$ \\
DP2 & $x_{7}^{1.0000}=$ constant & $80.28 \%$ \\
DP3 & $f_{1}^{0.3652} f_{2}^{1.0000} x_{5}^{-0.7699}=$ constant & $70.33 \%$ \\
DP4 & $x_{2}^{1.0000} x_{4}^{-0.1012} x_{5}^{-0.9360}=$ constant & $70.33 \%$ \\
DP5 & $f_{2}^{1.0000} x_{3}^{0.1293} x_{5}^{-0.8856}=$ constant & $70.12 \%$ \\
DP6 & $x_{2}^{1.0000} x_{5}^{-0.8748}=$ constant & $70.93 \%$ \\
DP7 & $f_{1}^{1.0000} x_{3}^{-0.2952} x_{5}^{-0.9675}=$ constant & $71.75 \%$ \\
DP8 & $f_{2}^{-0.6684} x_{2}^{1.0000} x_{5}^{-0.9887}=$ constant & $70.53 \%$ \\
DP9 & $x_{1}^{0.1166} x_{2}^{1.0000} x_{5}^{-0.9331}=$ constant & $72.15 \%$ \\
DP10 & $f_{2}^{1.0000} x_{1}^{0.2113} x_{5}^{-0.7592}=$ constant & $72.15 \%$ \\
DP11 & $f_{1}^{0.1161} x_{2}^{1.0000} x_{5}^{-0.8927}=$ constant & $71.14 \%$ \\
\hline
\end{tabular}

The objectives are to minimize the weight of the vehicle and the average rib deflection on the dummy. The complete formulation is provided in [6].

$$
\begin{array}{ll}
\text { Minimize } & f_{1}(\mathbf{x})=\text { Weight } \\
\text { Minimize } & f_{2}(\mathbf{x})=\left(D_{\text {ur }}+D_{m r}+D_{l r}\right) / 3 \\
\text { Subject to } & \text { Abdomen Load } \leq 1 \mathrm{kN}, \\
& \left\{V C_{\text {upper }}, V C_{\text {middle }}, V C_{\text {lower }}\right\} \leq 0.32 \mathrm{~m} / \mathrm{s}, \\
& \left.\left\{D_{\text {upper }}, D_{\text {middle }}, D_{\text {lower }}\right\} \text { (Rib deflections }\right) \leq 32 \mathrm{~mm}, \\
& F(\text { Pubic force } \leq 4 \mathrm{kN}, \\
& V_{M B P}(\text { Velocity of B-pillar midpoint }) \leq 9.9 \mathrm{~mm} / \mathrm{ms}, \\
& \left.V_{F D} \text { (Velocity of front door }\right) \leq 15.7 \mathrm{~mm} / \mathrm{ms} .
\end{array}
$$

The above problem is solved using NSGA-II and the obtained trade-off dataset is provided as input to the automated innovization algorithm. The obtained design principles and their significance values are shown in Table 1. Ten runs of the NSGA-II algorithm are performed and the generation-wise datasets for $N G E N=100$ generations are obtained. Using the procedure described in Section 2, the significance values are calculated for all 11 design principles at all generations and are plotted together as shown in Figure 1.

\subsection{Metal Cutting Problem}

In this problem [14], a steel bar is to be machined using a carbide tool of nose radius $r_{n}=0.8 \mathrm{~mm}$ on a lathe with $P^{\max }=10 \mathrm{~kW}$ rated motor to remove 219912 $\mathrm{mm}^{3}$ of material. A maximum cutting force of $F_{c}^{\max }=5000 \mathrm{~N}$ is allowed. The motor has a transmission efficiency $\eta$. The total operation time $\left(T_{p}\right)$ and the used tool life $(\xi)$ are to be minimized by optimizing the cutting speed $(v)$, the feed rate $(f)$ and the depth of cut $(a)$ while maintaining a surface roughness of 


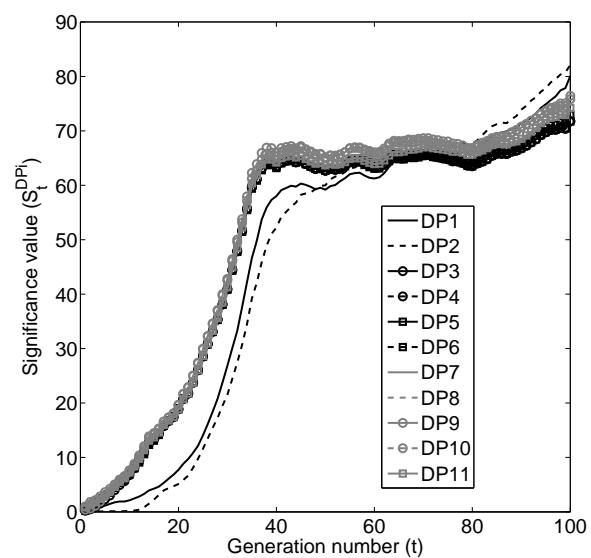

Fig. 1: Evolution of the 11 design principles shown in Table 1 for the car side impact problem.

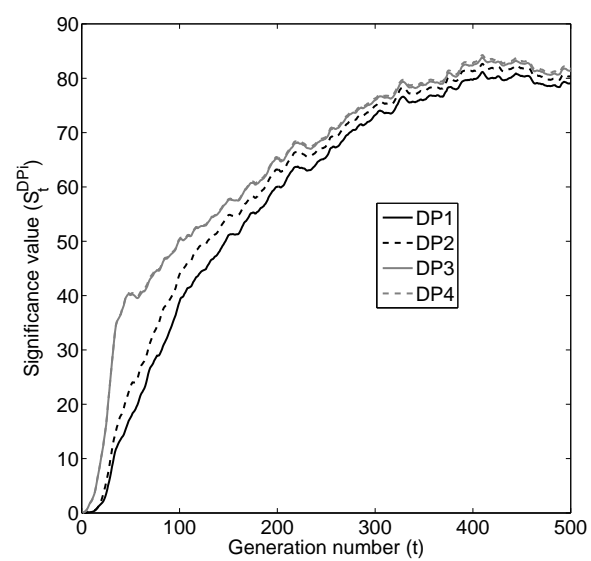

Fig. 2: Evolution of the four design principles shown in Table 2 for the metal cutting problem.

$R^{\max }=50 \mu m$. The problem is formulated as,

$$
\begin{array}{ll}
\text { Minimize } & f_{1}(\mathbf{x})=T_{p}(\mathbf{x}) \\
\text { Minimize } & f_{2}(\mathbf{x})=\xi(\mathbf{x}) \\
\text { Subject to } & P(\mathbf{x}) \leq \eta P^{\max } \\
& F_{c}(\mathbf{x}) \leq F_{c}^{\max } \\
& R(\mathbf{x}) \leq R^{\max } \\
& 250 \leq v \leq 400 \mathrm{~m} / \mathrm{min} \\
& 0.15 \leq f \leq 0.55 \mathrm{~mm} / \mathrm{rev} \\
& 0.5 \leq a \leq 6 \mathrm{~mm}
\end{array}
$$

where

$$
\begin{aligned}
& T_{p}(\mathbf{x})=0.15+219912\left(\frac{1+\frac{0.20}{T(\mathbf{x}}}{M R R(\mathbf{x})}\right)+0.05, \xi(\mathbf{x})=\frac{219912}{M R R(\mathbf{x}) T(\mathbf{x})} \times 100 \\
& T(\mathbf{x})=\frac{5.48 \times 10^{9}}{v^{3.46} f^{0.696} a^{0.460}}, \quad F_{c}(\mathbf{x})=\frac{6.56 \times 10^{3} f^{0.917} a^{1.10}}{v^{0.286}} \\
& P(\mathbf{x})=\frac{v F_{c}(\mathbf{x})}{60000}, \quad M R R(\mathbf{x})=1000 v f a, R(\mathbf{x})=\frac{125 f^{2}}{r_{n}} .
\end{aligned}
$$

The trade-off dataset obtained by solving Equation (3) using NSGA-II is used to generate the four design principles shown in Table 2 through automated innovization.

Again, ten runs of the NSGA-II algorithm are performed to obtain generationwise datasets for $N G E N=500$ generations. Figure 2 shows the plot of significance values versus generation number for all four design principles.

Both Figures 1 and 2 clearly show that design principles evolve in a gradual manner, as hypothesized in Section 1.1, slowly increasing in significance as the population converges close to the true Pareto-optimal front. However, the 
Table 2: Design principles for the metal cutting problem.

\begin{tabular}{|c|c|c|}
\hline Notation & Design principles & Significance \\
\hline DP1 & $f^{1.0000}=$ constant & $80.63 \%$ \\
DP2 & $\xi^{-0.3558} v^{1.0000}=$ constant & $80.85 \%$ \\
DP3 & $T_{p}^{0.9950} \xi^{-0.2712} v^{1.0000}=$ constant & $81.42 \%$ \\
DP4 & $T_{p}^{1.0000} v^{0.2391}=$ constant & $81.38 \%$ \\
\hline
\end{tabular}

secondary hypothesis that there may exist a hierarchy in the evolution of design principles cannot be verified in these examples. In fact, all design principles shown in the two figures evolve in exactly the same way for each problem. The design principles also appear very early during the optimization process. The reason for this is that these problems are only simplified versions of practical problems that are much more complex. In order to exhibit hierarchy during evolution, the design problem should be closer to the real-world, so that the multi-objective optimizer being used 'struggles' to build design principles during optimization thus bringing their hierarchy into the picture. In the next section, we reproduce the results obtained in [5] where the problem was shown to possess a hierarchy in the evolution of design principles.

\subsection{MEMS Resonator Design Problem}

The MEMS (MicroElectroMechanical System) component design problem involves the minimization of the power consumption $f_{1}$ (same as applied voltage, $V)$ and the minimization of the total area $f_{2}$ of the device. Figure 3 is a schematic of the MEMS model showing the 14 design variables. The complete problem formulation can be found in $[10,9]$. The problem is known to be highly non-linear in terms of the two objectives and involves 24 constraints (10 linear and 14 non-linear), making it rather difficult for NSGA-II to optimize and therefore to build design principles. Ten trade-off datasets are generated using NSGA-II. Their progress towards trade-off front at some specific generations is shown in Figure 4.

The design principles obtained through automated innovization are discussed in detail in [5]. Figure 5 shows the significance values for each of the 13 design principles at various generations. The evolution history shown in the figure reveals the time at which each principle started to evolve during the optimization process. The evolution is shown around $10 \%$ significance value. Clearly, a gradual evolution pattern of DPs can be seen along with the hierarchy (DP2, DP13, DP11, DP12, DP1, DP5, DP4, DP3, DP7, DP10, DP8, DP9, DP6). This information of some design principles evolving earlier than others may provide valuable knowledge about building a design from scratch in an optimal manner.

Discrete Variables in Evolution The MEMS resonator design involves a discrete variable $N_{c}$ representing the number of teeth on the rotor comb. Though no design principles involving this variable were obtained through automated 


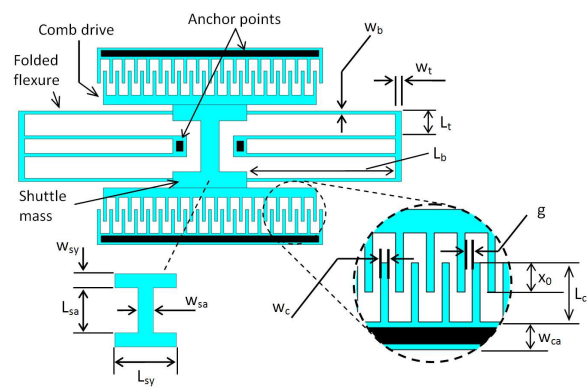

Fig. 3: MEMS resonator model showing the design variables.

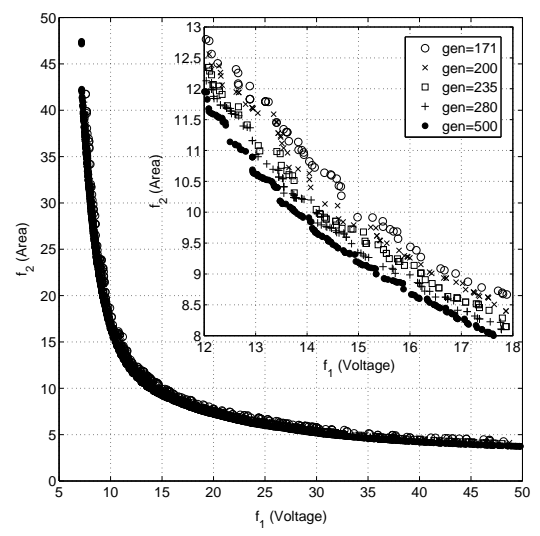

Fig. 4: Progress of solutions with generations towards trade-off front.

innovization, a simple manual innovization process reveals two design principles that do not fit the form in Equation (1) that relate $N_{c}$ to the objectives $f_{1}$ and $f_{2}$. These are,

$$
\begin{gathered}
\mathrm{B} 1 \equiv N_{c}-\frac{1.265 \times 10^{5}}{f_{1}^{3.829}} \text { and } \\
\mathrm{B} 2 \equiv 2.012 f_{2}-N_{c} .
\end{gathered}
$$

The significance values of B1 and B2 with generations are calculated as was done for the other design principles. Their evolution curves are also shown in Figure 5. B2 starts to evolve at around 130 generations and its evolution curve stays well before those of others. B1 appears in the population after B2 around the 160 generation mark and it too keeps evolving before the other design principles.

Coming back to human evolution, the anthropological description of the present day human too involves many discrete variables, like the number of eyes, limbs, fingers, backbones, etc. Taking the example of the human eye, it is now widely believed [13] that eyes initially appeared in the form of photoreceptive proteins that sense light and can only distinguish between bright and dark. Evidence for their existence can still be seen in certain green algae and unicellular organisms like euglenids [12]. The earliest eyes formed over millions of years as groups of photo-receptor cells came together and gradually depressed into what later became eye sockets. The number of such 'eyespots' was fixed very early on in the evolution of different species. In case of all ancestors of present day humans and related species, its value has always remained two. Though the current form of the human eye evolved only recently (relative to when life forms first appeared), the number of eyes was decided very early on in the evolution of man. Same is the case with number of limbs, fingers, etc.

Extending the analogy with human evolution to engineering design, it appears that whenever there are discrete variables involved, the evolution of optimal designs follows a path where design principles involving those discrete vari- 


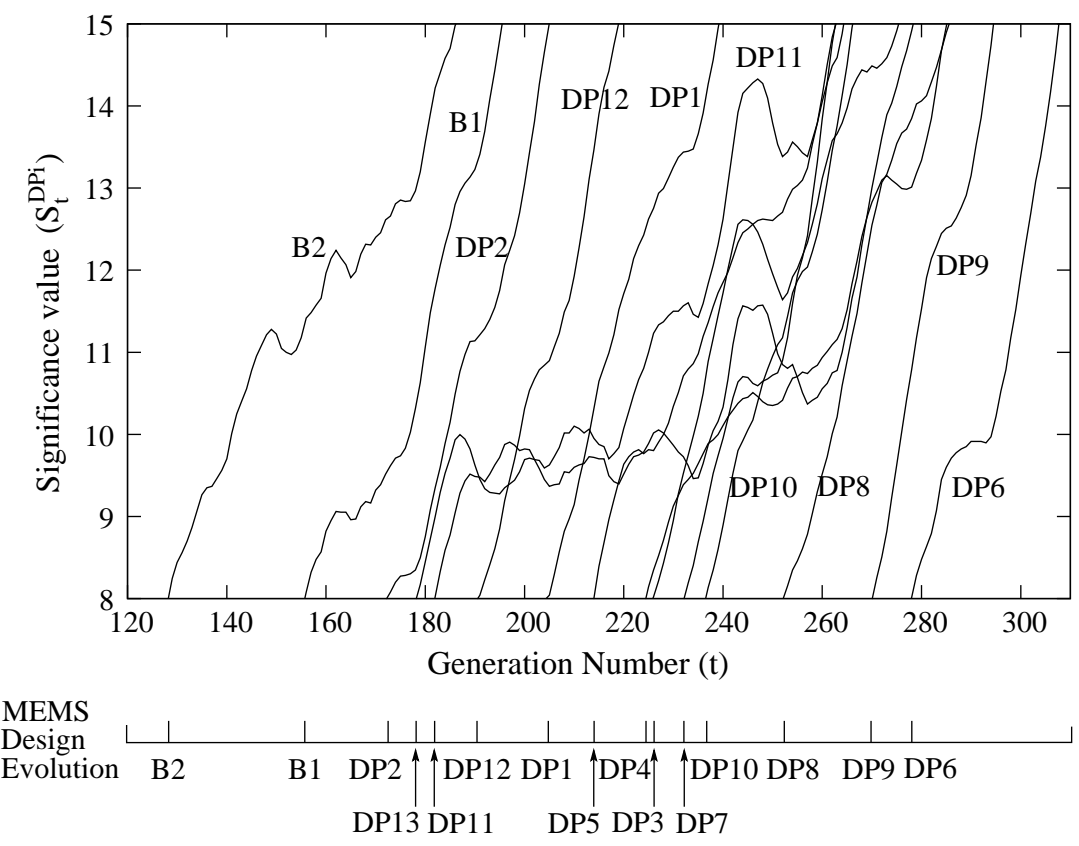

Fig. 5: Evolution of design principles for the MEMS design problem. The design principles evolve gradually but also maintain a hierarchy.

ables appear before any of the other design principles. This is at least the case in the MEMS resonator design problem as seen above. More problems need to be studied for evolution as described in this section to gather empirical evidence in support of this theory.

\section{Results II: Design Features through Superposition}

In certain engineering design problems where the trade-off designs can be represented schematically to show sufficient detail, the visual identification of optimal design features may prove to be more insightful than mathematical design principles obtained using automated innovization. In this section, we perform the temporal innovization task visually, since the nature of the problems used does not allow the generation of evolution plots.

Both problems used in this section concern structural topology optimization where the interest is in finding the topology (i.e. the distribution or layout of material inside) of a design domain subjected to loads and boundary conditions, so as to extremize one or more objectives. Recent research in topology optimization has been in the development of finite element based numerical methods as they can deal with complex topologies involving different types of materials [15]. Among numerical methods, especially popular are the so-called ISE (Isotropic Solid or Empty) topologies, in which blocks of finite elements (called ground 


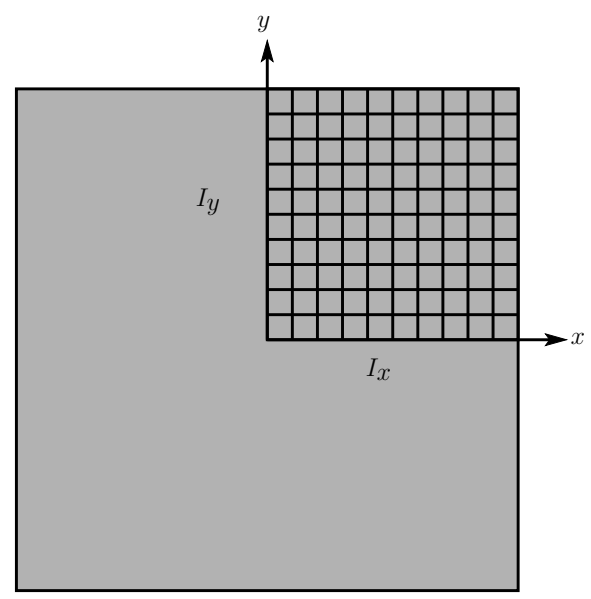

Fig. 6: Design domain for moment of inertia topology optimization problem.

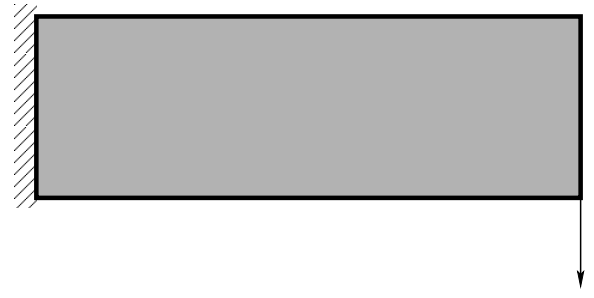

Fig. 7: Design domain for cantilever beam topology optimization problem.

elements) can either contain the given isotropic material (i.e. be solid) or contain no material at all (i.e. be empty). ISE topologies are represented using a 0-1 scheme. [16] argues that obtaining solutions with this scheme would be prohibitively expensive. The problem can be avoided by allowing ground elements to have intermediate densities and penalizing them using a power law [2].

\subsection{Moment of Inertia Problem}

The problem involves topology optimization of a square domain as shown in Figure 6 in order to, (i) maximize moment of inertia about the $x$-axis $\left(I_{x}\right)$ and, (ii) minimize moment of inertia about the $y$-axis $\left(I_{y}\right)$. The quarter problem is solved using a grid of size $10 \times 10$ as shown in Figure 6 and a $0-1$ representation, as described above. In the NSGA-II framework the 100 variables are represented using a binary string. For population members containing disconnected regions (identified after mirroring the $10 \times 10$ grid to full the whole domain), the clustering approach suggested in [3] is used to identify the largest cluster of cells with material and connected to the nearest axis. 100 random initial population members are evolved over 500 generations using the two-dimensional crossover operator [3] with $p_{c}=0.8$ and bit-wise mutation with $p_{m}=0.01$. The obtained trade-off front and a few designs are shown in Figure 8.

This problem is well-suited for extracting design features by superposition of solutions and studying their evolution. The procedure is very similar to that described in Section 2. The only difference is that after obtaining the set $\mathbf{P}_{t}$ of non-dominated solutions at the generation $t$, corresponding cell values from all solutions $\mathbf{P}_{t}$ are added to obtain a single matrix $\mathbf{M}_{t}$ of size $20 \times 20$. Thus, if a particular cell contains material (i.e. has a value of 1 ) in all non-dominated 


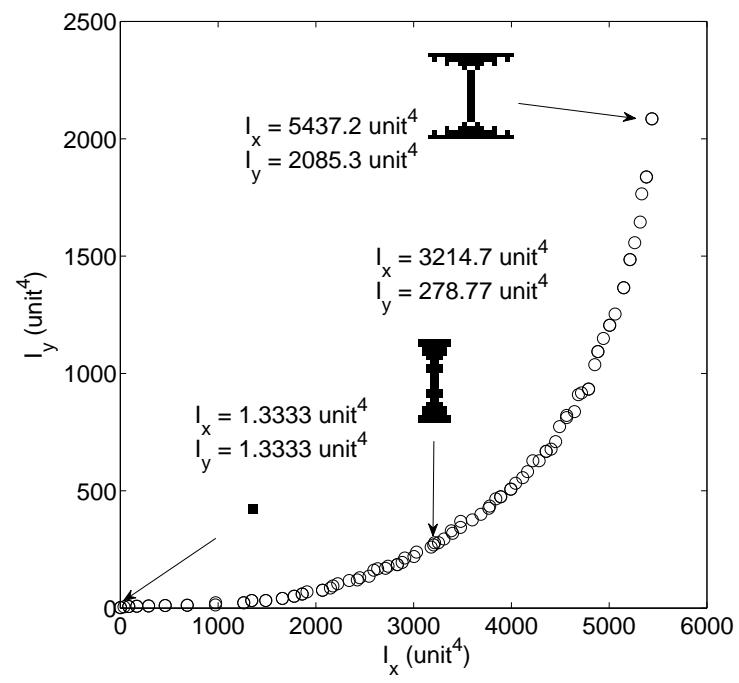

Fig. 8: Trade-off front for the moment of inertia topology optimization problem.

solutions of generation $t$, then its value in the resultant matrix becomes $\left|\mathbf{P}_{t}\right|$. The non-dominated solutions are therefore said to be superposed.

The status of design feature evolution at generation $t$ can be observed visually by the plotting the matrix $\mathbf{M}_{t}$, such that cells with the largest value are shown in black and those with the lowest value are shown in white. For the present problem, the evolution of design features is shown in Figure 9. It can be seen clearly that during the initial generations, solutions in which material is concentrated along the vertical centerline are non-dominated. Up until $t=30$ generations the material only spreads outwards from the vertical. Thereafter, evolution requires that the material should also be pushed outwards from the horizontal centerline (seen at $t=40$ ). The features at the corners of the domain, do not form until the later generations. Looking at $\mathbf{M}_{500}$ it can be said that, for maximizing $I_{x}$ and minimizing $I_{y}$, anything resembling an $I$-section would be close to Pareto-optimality. Though, in this case this property is intuitive, the study here revealed how exactly the $I$-section is formed during evolution.

\subsection{Cantilever Beam Problem}

In this problem, the topology of a cantilever beam carrying an end load of one unit is to be optimized within a rectangular domain as shown in Figure 7 for, (i) minimizing the compliance (or maximizing stiffness) and, (ii) minimizing the weight of the structure. Since the calculation of these objectives involves finite element simulations and the use 0-1 scheme may lead to indeterminate solutions in NSGA-II, the power-law approach of allowing intermediate densities suggested in [2] is employed. The intermediate densities are however not penalized as the present problem has two objectives and retaining such solutions may lead to 
better diversity. A grid of size $30 \times 10$ is imposed on the domain. The 300 variables are initialized for a population size of 400 and evolved using $\operatorname{SBX}\left(p_{c}=0.9\right)$ and polynomial mutation $\left(p_{m}=0.05\right)$ for 2000 generations. Finite element analysis is performed using [17] with the penalizing functions removed. The obtained trade-off front is shown in Figure 10 along with three different structures.

The evolution of design features can be obtained just as before. The only difference being that the cell values may contain any value in the range $[0,1]$ and $\mathbf{M}_{t}$ is of size $30 \times 10$. Figure 11 shows that the first feature to appear at $t=100$ is the strengthening of the structure near the lower support, indicated by darker regions. Cross members start appearing at around $t=200$ and the top right corner begins to disappear (whiter regions) around $t=300$ generations. As the generations progress, the above features become more and more prominent, while at $t=500$ holes start to form in the region between cross members. All features are eventually clearly seen at $t=2000$.

\section{Conclusions}

Temporal innovization refers to the evolution of the special properties that the Pareto-optimal solutions possess during the course of optimization using MOEAs. Based on the analogy with human evolution, it was hypothesized that design principles (like anthropological features of humans) evolve gradually over generations. It was also hypothesized that certain design principles will evolve earlier than others, if the presence of the former is essential for the development of the latter. This is also observed in the time-line of human evolution. For example, the development of limbs required a skeletal structure that can support the added weight. Thus, the development of vertebrae was essential to the appearance of the first limbs. While this kind of hierarchy is not observed for the simpler car side impact and metal cutting problems, it is clearly seen for the MEMS resonator design problem, which was difficult to be solved with NSGA-II.

Temporal innovization requires knowledge of the special properties. In our first set of problems, these properties were obtained through automated innovization. The growth of the design principles is studied with the help of evolution plots, which show the significance of different design principles as a function of the generation number of the MOEA. Our second set of problems concerned topology optimization, where solutions where represented in an unconventional manner. Therefore, instead of using automated innovization, we relied on the superposition of trade-off solutions at various generations, to observe the progress of design features possessed by the near Pareto-optimal solutions.

It may be argued that temporal innovization has limited practical uses. However, it is important to note that a temporal innovization study can reveal how optimizers build good designs from scratch. The most important aspects of the design are the ones that appear first during evolution. Such knowledge can lead to a deeper understanding of the problem at hand and enable the user to construct high-performing solutions simply by following certain thumb-rules of design. 


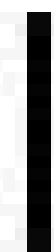

(a) $\mathbf{M}_{2}$

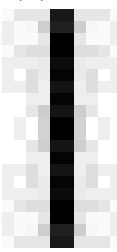

(d) $\mathbf{M}_{5}$

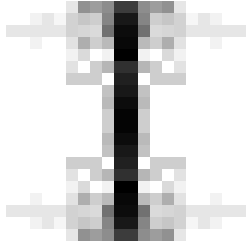

(g) $\mathbf{M}_{20}$

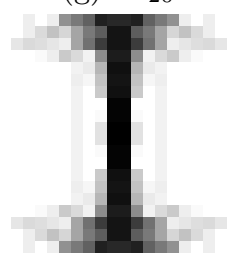

(j) $\mathbf{M}_{50}$

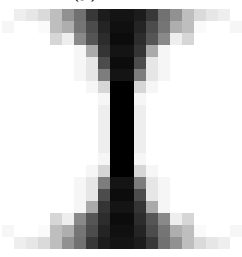

(m) $\mathbf{M}_{200}$

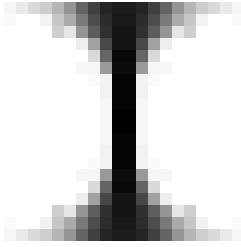

(p) $\mathbf{M}_{350}$

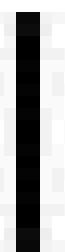

(b) $\mathbf{M}_{3}$

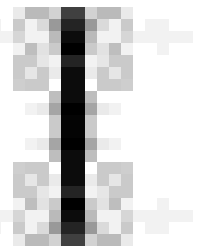

(e) $\mathbf{M}_{10}$

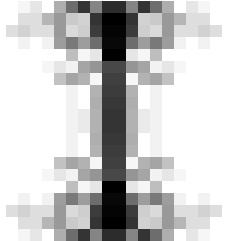

(h) $\mathbf{M}_{30}$

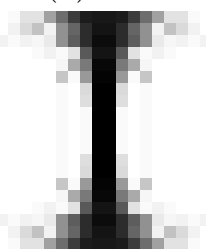

(k) $\mathbf{M}_{100}$

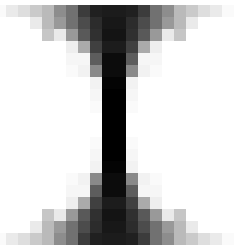

(n) $\mathbf{M}_{250}$

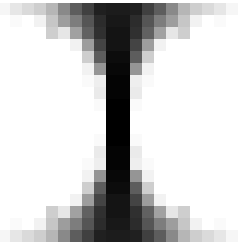

(q) $\mathbf{M}_{400}$

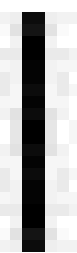

(c) $\mathbf{M}_{4}$

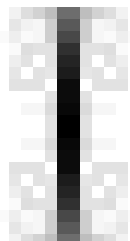

(f) $\mathbf{M}_{15}$

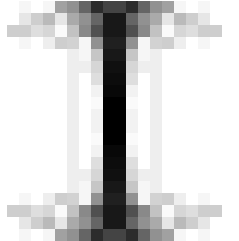

(i) $\mathbf{M}_{40}$

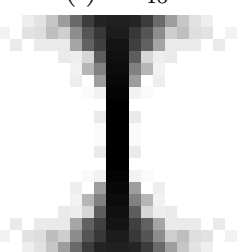

(l) $\mathbf{M}_{150}$

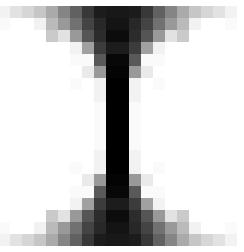

(o) $\mathbf{M}_{300}$

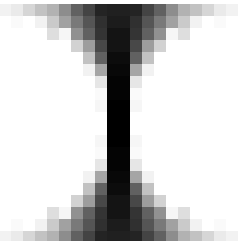

(r) $\mathbf{M}_{500}$

Fig. 9: Evolution of design features for moment of inertia topology optimization problem. $\mathbf{M}_{t}$ is obtained by superposing all non-dominated solutions from generation $t$. 


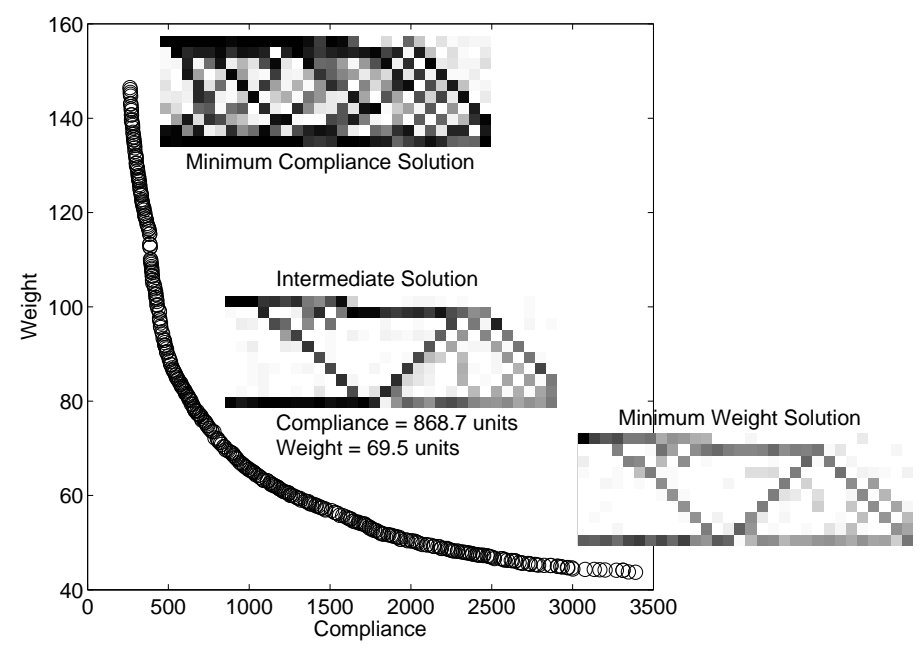

Fig. 10: Trade-off front for the cantilever beam topology optimization problem.

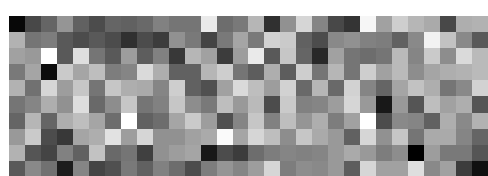

(a) $\mathbf{M}_{10}$

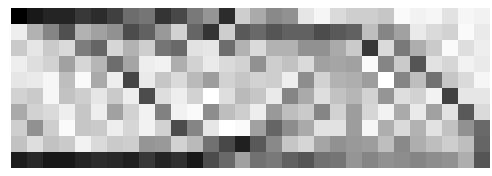

(c) $\mathbf{M}_{200}$

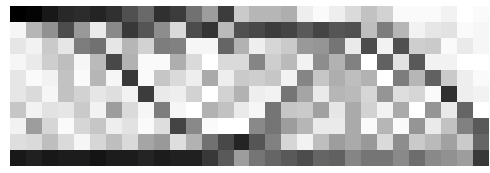

(e) $\mathbf{M}_{400}$

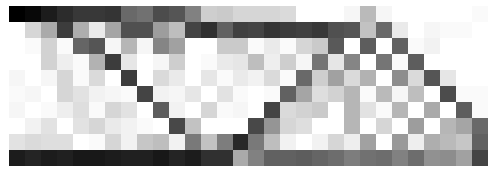

(g) $\mathbf{M}_{1000}$

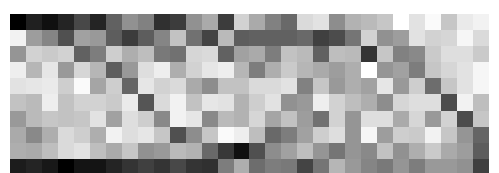

(b) $\mathbf{M}_{100}$

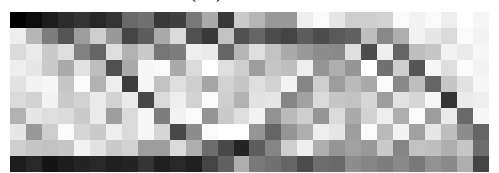

(d) $\mathbf{M}_{300}$

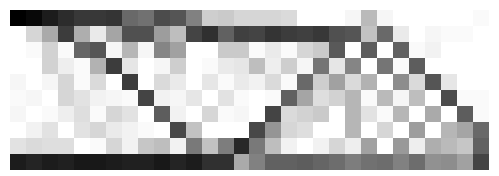

(f) $\mathbf{M}_{500}$

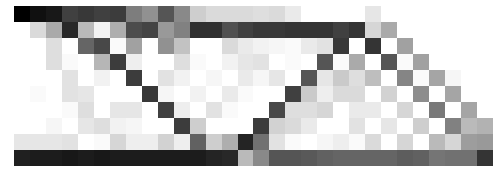

(h) $\mathbf{M}_{2000}$

Fig. 11: Evolution of design features for cantilever beam topology optimization problem. $\mathbf{M}_{t}$ is obtained by superposing all non-dominated solutions from generation $t$. 


\section{References}

1. Bandaru, S., Deb, K.: Towards automating the discovery of certain innovative design principles through a clustering-based optimization technique. Engineering Optimization 43(9), 911-941 (2011)

2. Bendsøe, M.: Optimal shape design as a material distribution problem. Structural and Multidisciplinary Optimization 1(4), 193-202 (1989)

3. Datta, D., Deb, K.: Design of optimum cross-sections for load-carrying members using multi-objective evolutionary algorithms. In: Proceedings of International Conference on Systemics, Cybernetics and Informatics. pp. 571-577. Proceedings of International Conference on Systemics, Cybernetics and Informatics (2005)

4. Deb, K., Agarwal, S., Pratap, A., Meyarivan, T.: A fast and elitist multi-objective genetic algorithm: NSGA-II. IEEE Transactions on Evolutionary Computation 6(2), 182-197 (2002)

5. Deb, K., Bandaru, S., Tutum, C.C.: Temporal evolution of design principles in engineering systems: Analogies with human evolution. In: Proceedings of the 12 th international conference on Parallel Problem Solving from Nature. pp. 1-10. PPSN'12, Springer-Verlag, Berlin, Heidelberg (2012)

6. Deb, K., Gupta, S., Daum, D., Branke, J., Mall, A., Padmanabhan, D.: Reliabilitybased optimization using evolutionary algorithms. IEEE Trans. on Evolutionary Computation 13(5), 1054-1074 (2009)

7. Deb, K., Srinivasan, A.: Innovization: Innovating design principles through optimization. In: Proceedings of the 8th annual conference on genetic and evolutionary computation, GECCO '06. pp. 1629-1636. New York: ACM (2006)

8. Deb, K., Bandaru, S., Greiner, D., Gaspar-Cunha, A., Tutum, C.C.: An integrated approach to automated innovization for discovering useful design principles: Case studies from engineering. Applied Soft Computing 15, 42-56 (2014)

9. Fedder, G., Iyer, S., Mukherjee, T.: Automated optimal synthesis of microresonators. In: Proceedings of the Ninth Int. Conf. Solid State Sens. Actuators. pp. 1109-1112. Chicago, IL (April 1997)

10. Fedder, G., Mukherjee, T.: Physical design for surface-micromachined MEMS. In: Proceedings of the Fifth ACM SIGDA Physical Design Workshop. Virginia, USA (April 1996)

11. Haeckel, E.: The evolution of man, Vol. 1. Kessinger Publishing (1879)

12. Kreimer, G.: The green algal eyespot apparatus: A primordial visual system and more? Current genetics 55(1), 19-43 (2009)

13. Land, M., Fernald, R.: The evolution of eyes. Annual review of neuroscience 15(1), 1-29 (1992)

14. Quiza Sardiñas, R., Rivas Santana, M., Alfonso Brindis, E.: Genetic algorithmbased multi-objective optimization of cutting parameters in turning processes. Engineering Applications of Artificial Intelligence 19(2), 127-133 (2006)

15. Rozvany, G.: Aims, scope, methods, history and unified terminology of computeraided topology optimization in structural mechanics. Structural and Multidisciplinary Optimization 21(2), 90-108 (2001)

16. Rozvany, G.: A critical review of established methods of structural topology optimization. Structural and Multidisciplinary Optimization 37(3), 217-237 (2009)

17. Sigmund, O.: A 99 line topology optimization code written in matlab. Structural and Multidisciplinary Optimization 21(2), 120-127 (2001) 